ORIGINAL

\title{
INDICADORES DE DISCAPACIDAD EN LA ENCUESTA DE DISCAPACIDADES, DEFICIENCIAS Y ESTADO DE SALUD
}

\author{
Julio Cabrero García \\ Departamento de Enfermería. Universidad de Alicante.
}

\section{RESUMEN}

Fundamento: La medida y significado de la discapacidad dependen de los indicadores que se utilicen. El objetivo de este estudio es examinar los indicadores de dependencia y discapacidad residual severa en dos niveles: en cada una de las 14 actividades de la vida diaria (AVD) seleccionadas, y como puntajes sumarios de recuento de actividades.

Métodos: Los datos proceden de la Encuesta de Discapacidades, Deficiencias y Estado de Salud (INE, 1999). La muestra se compone de los 8.779 registros correspondientes a las personas de 65 y más años con discapacidad en alguna de las 14 AVD seleccionadas. Se han creado tablas de proporciones y de contingencia, se han calculado estadísticos de asociación y concordancia, y se ha analizado la dimensionalidad de las medidas agregadas mediante el análisis factorial de información completa.

Resultados: Las principales discrepancias entre los indicadores se concentran en las actividades de movilidad (los coeficientes Kappa oscilaron entre 0,50 y 0,71 en actividades de movilidad y entre 0,73 y 0,87 en actividades domésticas y autocuidados). La discapacidad residual severa (indicador seleccionado por el INE) frente a la dependencia más incapacidad incluye a más personas discapacitadas en movilidad y excluye a más personas discapacitadas en actividades domésticas y autocuidados. Dependencia más incapacidad es el indicador con mayor validez interna como medida agregada de discapacidad (dos autovalores: 8,37 y 1,$91 ; 56,3 \%$ varianza del primer factor, saturaciones del primer factor entre 0,58 y 0,87 ).

Conclusiones: La dependencia más la incapacidad es un indicador más valido que la discapacidad residual severa como indicador principal de discapacidad.

Palabras clave: Valoración de la discapacidad. Actividades de la vida diaria. Cuidados de larga duración. Personas mayores.

Correspondencia:

Julio Cabrero García

Departamento de Enfermería

Universidad de Alicante

Campus de San Vicente del Raspeig

Apdo. 99 E-03080

Correo electrónico: julio.cabrero@ua.es

\section{ABSTRACT}

\section{Disability indicators in the Spanish Disability, Impairment and Health Survey}

Background: The measurement and meaning of disability depend on the indicators used. In this study the indicators of dependency and severe residual disability are examined on two levels: in each of the 14 selected activities of daily living (ADL) and also as a summary score of the activities count.

Methods: The data are extracted of the Disability, Impairment and Health Survey (INE, 1999). The sample is composed of 8.779 entries corresponding to people over 65 years old with disability in some of the 14 selected ADL. Tables of proportions and contingency have been elaborated, statistics of association and agreement has been calculated and the dimensionality of the aggregated measures had been analyzed through the full information factor analysis.

Results: The main discrepancies between the indicators are concentrated on the mobility activities (kappa coefficients ranged between 0,50 to 0,75 for mobility activities and between 0,73 to 0,87 for domestic activities and self-care). Severe residual disability (indicator selected by the National Institute of Statistics) compared to dependency more inability includes more mobility disabled people and excludes more disabled people in domestic activities and self-care. Dependency more inability is the indicator with the highest validity as a summary measure of disability (two eigenvalues: 8,37 and 1,91, the first factor accounted for $56,3 \%$ of the variance, the saturations of the first factor ranged between 0,58 to 0,87 ).

Conclusions: Dependency more inability is more valid than severe residual disability as main indicator of disability.

Keywords: Disability evaluation. Activities of daily living. Long-term care. Aged. Aged, 80 and over. 


\section{INTRODUCCIÓN}

La discapacidad en las personas mayores es el predictor principal, después de la edad, de numerosas consecuencias importantes, entre las que se incluye la mortalidad, el declinar de la capacidad funcional, la incidencia de enfermedades agudas, caídas y lesiones, y la utilización de servicios sanitarios e institucionalización ${ }^{1}$. A escala poblacional las medidas de discapacidad se utilizan para monitorizar y valorar la salud pública ${ }^{2}$, ya que permiten describir tendencias en la salud general de la población mayor ${ }^{3}$ y planificar los cuidados de larga duración y los programas de servicios sociales ${ }^{4,5}$. En España el instrumento más reciente e importante para examinar el fenómeno de la discapacidad es la Encuesta de Discapacidades, Deficiencias y Estado de Salud (EDDES), realizada por el INE6.

La discapacidad se evalúa, generalmente, mediante auto-informes de dificultad o de necesidad de ayuda en actividades de la vida diaria (AVD) $)^{1,3}$. Sin embargo, las estimaciones de prevalencia y de demanda potencial de servicios varían según el indicador de discapacidad empleado: los indicadores basados en la dependencia, esto es, la recepción o necesidad de ayuda personal ${ }^{7}$ clasifican un número menor de personas como discapacitadas que los indicadores basados en la dificultad y son mejores indicadores de demanda potencial de asistencia ${ }^{8-10}$. Consecuentemente, la dependencia es el indicador más usado en las encuestas poblacionales para planificar los cuidados de larga duración de las personas discapaci$\operatorname{tadas}^{11}$. Una alternativa a la dependencia es la discapacidad residual, es decir, la dificultad usual con ayuda personal o técnica si se recibe ${ }^{3}$. Éste es un indicador de concepción y uso más reciente que la dependencia, siendo escasos los estudios que comparen ambos indicadores ${ }^{10}$. Sin embargo, el INE ${ }^{12}$, el IMSERSO ${ }^{13}$ y otros que emplean la $\operatorname{EDDES}^{14,15,16}$ se han decantado por usar la discapacidad residual como indicador de discapacidad frente a la dependencia $\mathrm{u}$ otros. Estrictamente, estas fuentes ${ }^{12-16}$ seleccionan los dos grados superiores de discapacidad residual, es decir, discapacidad residual severa. Así, en España más de un millón de personas mayores de 64 años sufren discapacidad residual severa en AVD $^{12}$.

Los objetivos de esta investigación con los datos de la EDDES de las personas mayores de 64 años son comparar los indicadores de dependencia y discapacidad residual severa en cuanto a la estimación del porcentaje de discapacitados en cada una de las 14 AVD, así como su composición respecto a la ayuda recibida y a la presencia de incapacidad; y examinar cuál de estos indicadores tiene mayor validez interna como medida agregada (recuento de discapacidades) de severidad de la discapacidad.

\section{MATERIAL Y MÉTODOS}

Fuente de los datos: Los datos proceden de la EDDES, una gran encuesta multifásica de cobertura nacional de más de 70.000 hogares. En una primera fase se captó a los miembros del hogar con alguna discapacidad (o limitación, en el caso de los menores de 6 años). Se definió discapacidad como toda limitación grave de la actividad (aunque estuviera superada con el uso de ayudas técnicas externas) que afectara o pudiera afectar durante más de un año y con origen en una deficiencia o en procesos degenerativos asociados a la edad. Esta definición es asimilable al concepto de discapacidad subyacente $^{3}$. En una segunda fase se entrevistó a las personas identificadas como discapacitadas (discapacidad subyacente) en la primera fase. Se han usado dos ficheros: el de "personas", que contiene las variables sociodemográficas de los 
218.185 sujetos encuestados de los que 37.582 tenían 65 o más años de edad, y el fichero "todo discapacidades", que contiene las variables relativas a las características de las discapacidades de las 20.224 personas de 6 o más años de edad que tienen una o más de las 36 discapacidades incluidas en la encuesta. De las 36 discapacidades 15 son AVD y el resto son deficiencias o limitaciones ${ }^{11}$ en términos del "proceso de discapacidad"7. La discapacidad en las AVD reúne a la gran mayoría de las personas que reciben ayuda $^{11}$, por lo que estas actividades constituyen la base primordial para estudiar la dependencia y la severidad de la discapacidad. La EDDES las agrupa en cuatro tipos: autocuidados (lavarse, continencia e ir al servicio, vestirse, comer y beber), movilidad dentro de casa (cambios y mantenimiento de las posiciones del cuerpo, levantarse, acostarse, permanecer de pie o sentado, y desplazamiento dentro del hogar), actividades domésticas (hacer las compras y controlar los suministros y servicios, cuidarse de las comidas, de la limpieza y planchado de la ropa, de la limpieza y mantenimiento de la casa, y del bienestar de los demás miembros de la familia) y movilidad exterior (deambular sin medio de transporte, desplazarse en transportes públicos y conducir el vehículo propio). Los dos primeros grupos son AVD básicas (ABVD) y los dos últimos AVD instrumentales (AIVD). En este estudio se han seleccionado sólo las AVD, y de éstas se ha excluido la de conducir el vehículo, dada su escasa relevancia en las personas mayores. La muestra analizada se compone de las 8.779 personas de 65 y más años de edad que tienen alguna discapacidad subyacente en las 14 AVD.

Medidas: Se han empleado variables de medida de la EDDES y se han creado otras a partir de éstas. Respecto a las primeras se han seleccionado las siguientes. Discapacidad subyacente, que clasifica a las personas como discapacitadas o no en cada una de las actividades encuestadas; establece además el umbral inferior para todos los indicadores de discapacidad. En la segunda fase la EDDES preguntó a las personas con discapacidad subyacente (identificadas en la primera) por el tipo de ayuda que recibían: ayudas técnicas, ayudas personales, ayudas técnicas y personales y sin ayuda; por el tipo de ayuda que habían solicitado y no recibían (las mismas categorías que la anterior); y por el grado de discapacidad residual $^{7}$, es decir, la dificultad considerando la ayuda que recibían, si tal fuera el caso: dificultad moderada, dificultad grave e incapacidad para hacer la actividad. A partir de estas variables de medida (discapacidad subyacente, tipos de ayuda, tipos de solicitud de ayuda insatisfecha y discapacidad residual) se han creado las siguientes variables binarias: dependencia (personas que reciben ayuda frente a las que no); incapacidad (personas que no pueden realizar la actividad frente a las que sí); dependencia más incapacidad (personas dependientes más aquellas con incapacidad frente al resto); solicitud insatisfecha de ayuda personal (personas que han solicitado ayuda personal y no la han recibido frente al resto); solicitud insatisfecha de ayudas técnicas (personas que han solicitado ayuda técnica y no la han recibido frente al resto) y discapacidad residual severa (personas con discapacidad residual grave o incapaces de realizar la actividad frente al resto. Éste es el indicador de severidad de la discapacidad empleado por el INE y el IMSERSO). Se han creado, por último, variables sumarias de recuento de actividades con los dos indicadores de dependencia y con discapacidad residual severa.

Análisis de datos: Los micro-datos, en texto plano, fueron procesados con el editor del paquete estadístico $\operatorname{SPSS}^{17}$. Se han calculado porcentajes y tablas de contingencia. Se ha calculado el índice de 
Tabla 1

Porcentajes de discapacidades según diversos indicadores en personas de 65 y más años $(\mathbf{n}=37.582)$

\begin{tabular}{|c|c|c|c|c|c|c|c|}
\hline $\begin{array}{l}\text { Actividades de la vida } \\
\text { diaria }\end{array}$ & $\begin{array}{l}\text { Discapacacidad } \\
\text { subyacente }\end{array}$ & Dependencia & $\begin{array}{c}\text { Dependencia + } \\
\text { incapacidad }\end{array}$ & $\begin{array}{l}\text { Dis. residual } \\
\text { severa }\end{array}$ & Incapacidad & SIA personal & SIA técnica \\
\hline \multicolumn{8}{|l|}{ M. interior } \\
\hline Posiciones & $6,84(29,0)$ & $3,96(16,8)$ & $\begin{array}{c}\text { Idem } \\
\text { dependencia }\end{array}$ & $4,32(18,3)$ & $0,97(4,1)$ & 7,1 & 1,8 \\
\hline Transferencia* & $10,45(44,3)$ & $5,50(23,3)$ & $5,90(25,0)$ & $6,14(26,0)$ & $1,18(5,0)$ & 6,2 & 1,7 \\
\hline Andar dentro & $9,0(38,5)$ & $4,44(18,8)$ & $4,91(20,8)$ & $5,48(23,2)$ & $1,18(5,0)$ & 6,1 & 2,2 \\
\hline \multicolumn{8}{|l|}{ Autocuidados } \\
\hline Aseo & $8,09(34,3)$ & $7,41(31,4)$ & $\begin{array}{c}\text { Idem } \\
\text { dependencia }\end{array}$ & $5,71(24,2)$ & $1,68(7,1)$ & 7,6 & 0,6 \\
\hline Uso WC & $3,99(16,9)$ & $3,47(14,7)$ & $\begin{array}{c}\text { Idem } \\
\text { dependencia }\end{array}$ & $3,23(13,7)$ & $1,13(4,8)$ & 9,4 & 1,4 \\
\hline Vestirse & $6,75(28,6)$ & $6,16(26,1)$ & $\begin{array}{c}\text { Idem } \\
\text { dependencia }\end{array}$ & $4,91(20,8)$ & $1,56(6,6)$ & 8,1 & 0,4 \\
\hline Comer, beber & $2,48(10,5)$ & $2,27(9,60)$ & $\begin{array}{c}\text { Idem } \\
\text { dependencia }\end{array}$ & $1,98(8,4)$ & $0,68(2,9)$ & 9,8 & 0,4 \\
\hline \multicolumn{8}{|l|}{ M. exterior } \\
\hline Andar fuera & $17,53(74,3)$ & $7,81(33,1)$ & $9,67(41,41)$ & $10,79(45,7)$ & $2,41(10,2)$ & 3,8 & 1,4 \\
\hline Uso de transporte & $18,55(78,6)$ & $8,00(33,9)$ & $11,17(47,8)$ & $12,00(51,0)$ & $3,68(15,6)$ & 2,1 & 1,0 \\
\hline \multicolumn{8}{|l|}{ A. domésticas } \\
\hline Comprar & $13,42(56,9)$ & $11,89(50,4)$ & $\begin{array}{c}\text { Idem } \\
\text { dependencia }\end{array}$ & $11,12(47,1)$ & $7,17(30,4)$ & 5,1 & 0,3 \\
\hline Preparar comida & $9,63(40,8)$ & $8,59(36,4)$ & $\begin{array}{c}\text { Idem } \\
\text { dependencia }\end{array}$ & $7,93(33,6)$ & $4,96(21,0)$ & 5,5 & 0,3 \\
\hline Lavar, planchar & $11,78(49,9)$ & $10,36(43,9)$ & $\begin{array}{c}\text { Idem } \\
\text { dependencia }\end{array}$ & $9,51(40,3)$ & $5,62(23,8)$ & 5,6 & 0,3 \\
\hline Cuidados de casa & $14,14(59,9)$ & $12,2(51,7)$ & $\begin{array}{c}\text { Idem } \\
\text { dependencia }\end{array}$ & $10,97(46,5)$ & $6,09(2,8)$ & 6,0 & 0,3 \\
\hline Cuidar a otros & $9,16(38,8)$ & $7,9(33,5)$ & $\begin{array}{c}\text { Idem } \\
\text { dependencia }\end{array}$ & $7,81(33,1)$ & $5,38(22,8)$ & 4,0 & 0,3 \\
\hline
\end{tabular}

Notas: Los porcentajes se han calculado usando como denominador el número total de personas de 65 y más años de edad encuestadas en la EDDES. Entre paréntesis son los porcentajes calculados usando como denominador el número de personas con alguna discapacidad en actividades de la vida diaria $(n=8.779)$. SIA es solicitud insatisfecha de ayuda. Los siete indicadores se definen en el texto, en el apartado de Medidas.

* Transferencia es acostarse, levantarse, permanecer de pie o sentado.

Kappa para medir la concordancia entre los dos indicadores de dependencia y discapacidad residual severa, considerando los factores que pueden afectar a su magnitud $^{18}$ : índices de prevalencia y sesgo, e independencia entre observaciones (el indicador de dependencia más incapacidad comparte con discapacidad residual severa, por definición, a las personas con incapacidad; esto sucede en cuatro actividades de movilidad, en el resto la concordancia es empírica (tabla 1) no un artefacto de medida); y la correlación tetracórica para medir la asociación entre los indicadores binarios de solicitud insatisfecha de ayuda y de discapacidad. La correlación tetracórica es mejor opción que la correlación producto de momento phi, ya que puede lograr los valores +1 y 1 cuando las proporciones marginales difieren; la correlación phi, en cambio, no puede lograrlos ${ }^{19}$. La dimensionalidad de las 14 AVD se ha examinado con el análisis factorial de información completa (AFIC), un procedimiento no lineal basado en la teoría de respuesta al item (su solución unidimensional es equivalente a un modelo de dos parámetros) ${ }^{20}$. El AFIC es 
una técnica más adecuada para evaluar la dimensionalidad de ítems binarios que el análisis factorial lineal ${ }^{21}$. Para evitar aumentar artificialmente la covariación entre los ítems, las muestras de análisis han incluido sólo a las personas discapacitadas en AVD según el indicador empleado. En la valoración del ajuste de las soluciones unidimensionales se han empleado criterios clásicos como el porcentaje de varianza explicada y la razón entre los dos primeros autovalores ${ }^{22}$. El AFIC se ha implementado con TESTFACT $^{23}$. No se incorporan en el análisis de datos los pesos de muestreo, de forma que se aludirá a porcentajes y no a prevalencias.

\section{RESULTADOS}

En la EDDES se encuestó a 37.582 personas de 65 y más años de edad, de las que $21.316(56,72 \%)$ fueron mujeres. Del total de personas mayores encuestadas 8.779 personas $(23,36 \%)$ tenían discapacidad subyacente en alguna de las 14 AVD. Entre las personas mayores con alguna discapacidad subyacente en AVD $6.011(68,5 \%)$ eran mujeres y 3.248 (37\%) tenían más de 80 años, siendo la edad media 77,94 $(\mathrm{dt}=7,8)$. En la tabla 1 se muestra el porcentaje de personas discapacitadas según diversos indicadores de discapacidad en las 14 AVD. Dependencia y dependencia más incapacidad dan valores idénticos salvo en las actividades de movilidad, principalmente en la movilidad exterior: todas las personas con incapacidad en autocuidados y actividades domésticas son dependientes, pero no sucede así en movilidad, de forma que no incluir la incapacidad en el indicador de dependencia subestima la discapacidad en las actividades de movilidad. Discapacidad subyacente es, por motivos de diseño de la EDDES, el indicador con los porcentajes más elevados de discapacidad. Las diferencias con los indicadores de de- pendencia y discapacidad residual severa se acentúan en las actividades de movilidad. Discapacidad residual severa identifica más personas discapacitadas en movilidad que dependencia y menos en las actividades domésticas y en autocuidados. Respecto a los indicadores auxiliares, son notables los pequeños porcentajes de solicitud insatisfecha de ayuda personal, y aún menores los de solicitud insatisfecha de ayudas técnicas. La proporción de solicitud insatisfecha de ayuda se ordena según la dificultad de la actividad: autocuidados, movilidad interior, actividades domésticas y movilidad exterior. En el indicador de incapacidad se observa un gran salto porcentual de las actividades domésticas a los autocuidados. La distancia entre las actividades de movilidad interior y exterior es inferior, pero también evidente. Además, la mayoría de las personas incapaces en movilidad exterior no reciben ayuda.

Las distribuciones marginales de la ayuda y de la discapacidad residual y su distribución conjunta, aparecen en las tablas 2 y 3 . Los porcentajes de ayuda sólo técnica son muy apreciables en las actividades de movilidad, pero son mínimos en el resto de las AVD; el mismo patrón se da para la categoría sin ayuda. Sucede al contrario para las dos categorías que incluyen ayuda personal: son muy elevados en autocuidados y actividades domésticas y sensiblemente inferiores en movilidad. En definitiva, el patrón de la ayuda en la movilidad es diferente al del resto de las AVD: entre la no discapacidad subyacente y la dependencia son probables la discapacidad subyacente sin ayuda y la discapacidad subyacente con ayuda sólo técnica; estos dos pasos intermedios son infrecuentes en el resto de AVD. La discapacidad residual distingue también a las actividades de movilidad de las domésticas y los autocuidados: en las primeras hay más discapacidad residual moderada y en las segundas hay más discapacidad 
Tabla 2

Discapacidad residual y tipos de ayuda en ABVD: movilidad interior y autocuidados. Porcentajes

\begin{tabular}{|c|c|c|c|c|c|}
\hline Discapacidad residual & $\%$ & Sin ayuda & Técnica & Personal & Pers. y téc. \\
\hline \multicolumn{6}{|l|}{ Posiciones $(n=2.484)$} \\
\hline Moderada & 35,2 & 18,8 & 5 & $8,7^{\mathrm{a}}$ & $2,8^{\mathrm{a}}$ \\
\hline Grave & 50,4 & $12^{\mathrm{b}}$ & $5,5^{\mathrm{b}}$ & 23,8 & 9,2 \\
\hline \multirow[t]{2}{*}{ Incapaz } & 14,3 & 0 & 0 & 11,6 & 2,7 \\
\hline & & 30,8 & 10,5 & 44,1 & 14,7 \\
\hline \multicolumn{6}{|l|}{ Transferencia $n=3.782$ ) } \\
\hline Moderada & 40,0 & 18,1 & 7 & $11,2^{\mathrm{a}}$ & $3,7^{\mathrm{a}}$ \\
\hline Grave & 48,5 & $10,8^{\mathrm{b}}$ & $6,8^{\mathrm{b}}$ & 21,7 & 9,1 \\
\hline \multirow[t]{2}{*}{ Incapaz } & 11,5 & 3,9 & 0 & 5,1 & 2,5 \\
\hline & & 32,8 & 13,8 & 38,1 & 15,4 \\
\hline \multicolumn{6}{|l|}{ Andar dentro $(n=3.280)$} \\
\hline Moderada & 38,2 & 12,2 & 14,1 & $7^{\mathrm{a}}$ & $4,9^{\mathrm{a}}$ \\
\hline Grave & 48,5 & $7^{\mathrm{b}}$ & $11,6^{\mathrm{b}}$ & 15,9 & 14,1 \\
\hline \multirow[t]{2}{*}{ Incapaz } & 13,3 & 5,2 & 0 & 4,2 & 3,9 \\
\hline & & 24,3 & 25,7 & 27,1 & 22,9 \\
\hline \multicolumn{6}{|l|}{ Aseo $(n=2.907)$} \\
\hline Moderada & 27,6 & 2,7 & 0,6 & $22,9^{\mathrm{a}}$ & $1,4^{\mathrm{a}}$ \\
\hline Grave & 51,0 & $3,4^{b}$ & $0,6^{\mathrm{b}}$ & 43,8 & 3,2 \\
\hline \multirow[t]{2}{*}{ Incapaz } & 21,4 & 0 & 0 & 19,8 & 1,6 \\
\hline & & 6,1 & 1,2 & 86,5 & 6,2 \\
\hline \multicolumn{6}{|l|}{ Uso WC $(\mathrm{n}=1.448)$} \\
\hline Moderada & 17,3 & 3,2 & 0,8 & $11,5^{\mathrm{a}}$ & $1,9^{\mathrm{a}}$ \\
\hline Grave & 53,5 & $4,9^{b}$ & $2,6^{\mathrm{b}}$ & 36,4 & 9,5 \\
\hline \multirow[t]{2}{*}{ Incapaz } & 29,2 & 0 & 0,6 & 23,1 & 5,5 \\
\hline & & 8,1 & 4,0 & 70,9 & 17,0 \\
\hline \multicolumn{6}{|l|}{ Vestirse $(\mathrm{n}=2.434)$} \\
\hline Moderada & 25,5 & 3,3 & 0,2 & $21,2^{\mathrm{a}}$ & $0,7^{\mathrm{a}}$ \\
\hline Grave & 50,7 & $3,7^{\mathrm{b}}$ & $0,4^{\mathrm{b}}$ & 43,6 & 2,9 \\
\hline \multirow[t]{2}{*}{ Incapaz } & 23,8 & 0 & 0 & 22,3 & 1,5 \\
\hline & & 7,1 & 0,6 & 87,2 & 5,2 \\
\hline \multicolumn{6}{|l|}{ Comer y Beber $(n=907)$} \\
\hline Moderada & 19,1 & 3,2 & 0,2 & $15,3^{\mathrm{a}}$ & $0,3^{\mathrm{a}}$ \\
\hline Grave & 52,7 & $4^{\mathrm{b}}$ & $0,3^{\mathrm{b}}$ & 46,6 & 1,8 \\
\hline \multirow[t]{2}{*}{ Incapaz } & 28,2 & 0 & 0 & 25,9 & 2,3 \\
\hline & & 7,2 & 0,6 & 87,9 & 4,4 \\
\hline
\end{tabular}

Los porcentajes se han calculado tomando como denominador el número de personas discapacitadas en cada actividad.

a: Porcentaje de personas excluidas con el criterio de discapacidad residual severa (grave o incapaz) respecto a dependencia más incapacidad

b: Porcentaje de personas excluidas con el criterio de dependencia más incapacidad respecto a discapacidad residual severa (grave o incapaz).

residual severa. Las clasificaciones cruzadas entre tipos de ayuda y discapacidad residual permiten examinar las implicaciones de elegir entre discapacidad residual severa (los dos grados superiores de discapacidad residual) y dependencia más incapacidad (ayuda personal e incapaci- dad) a partir de los valores con superíndices en las tablas 2 y 3 . Por ejemplo, si la discapacidad en la actividad de aseo personal se determinara según el indicador de discapacidad residual severa se excluiría a un 24,3\% $(22,9+1.4)$ de personas dependientes en esa actividad (tabla 2). Si, 
Tabla 3

Discapacidad residual y tipos de ayuda en AIVD: movilidad exterior y actividades domésticas. Porcentajes

\begin{tabular}{|c|c|c|c|c|c|}
\hline Discapacidad residual & $\%$ & Sin ayuda & Técnica & Personal & Pers. y téc. \\
\hline \multicolumn{6}{|l|}{ Andar fuera $(\mathrm{n}=6.313)$} \\
\hline Moderada & 37,2 & 12,4 & 11 & $9,4_{\mathrm{a}}$ & $4,4^{\mathrm{a}}$ \\
\hline Grave & 48,6 & $10,7^{\mathrm{b}}$ & $9,3^{\mathrm{b}}$ & 18,3 & 10,5 \\
\hline \multirow[t]{2}{*}{ Incapaz } & 14,1 & 11,5 & 0 & 0 & 2,7 \\
\hline & & 34,5 & 20,3 & 27,7 & 17,6 \\
\hline \multicolumn{6}{|l|}{ Uso transporte $(\mathrm{n}=6.730)$} \\
\hline Moderada & 34,1 & 13,6 & 6,5 & $10,5^{\mathrm{a}}$ & $3,5^{\mathrm{a}}$ \\
\hline Grave & 45,6 & $12,1^{\mathrm{b}}$ & $6,3^{\mathrm{b}}$ & 19,3 & 7,9 \\
\hline \multirow[t]{2}{*}{ Incapaz } & 20,3 & 18,1 & 0 & 0 & 2,2 \\
\hline & & 43,8 & 12,8 & 29,8 & 13,6 \\
\hline \multicolumn{6}{|l|}{ Comprar $(n=4.835)$} \\
\hline Moderada & 15,2 & 2,9 & 0,5 & $11^{\mathrm{a}}$ & $0,9^{\mathrm{a}}$ \\
\hline Grave & 29,6 & $5,9^{\mathrm{b}}$ & $0,6^{\mathrm{b}}$ & 20,4 & 2,7 \\
\hline \multirow[t]{2}{*}{ Incapaz } & 55,1 & 0 & 0 & 55,1 & 0 \\
\hline & & 8,8 & 1,1 & 86,5 & 3,6 \\
\hline \multicolumn{6}{|l|}{ Preparar comidas $(\mathrm{n}=3.476)$} \\
\hline Moderada & 15,5 & 3 & 0,1 & $11,6^{\mathrm{a}}$ & $0,8^{\mathrm{a}}$ \\
\hline Grave & 31,5 & $5,7^{\mathrm{b}}$ & $0,5^{\mathrm{b}}$ & 23,1 & 2,2 \\
\hline \multirow[t]{2}{*}{ Incapaz } & 53,0 & 0 & 0 & 53 & 0 \\
\hline & & 8,7 & 0,6 & 87,7 & 3,0 \\
\hline \multicolumn{6}{|l|}{ Lavar y Planchar $(n=4.242)$} \\
\hline Moderada & 17,1 & 3,4 & 0,4 & $12,5^{\mathrm{a}}$ & $0,8^{\mathrm{a}}$ \\
\hline Grave & 33,7 & $6,1^{\mathrm{b}}$ & $0,6^{\mathrm{b}}$ & 24,4 & 2,7 \\
\hline \multirow[t]{2}{*}{ Incapaz } & 49,2 & 0 & 0 & 49,2 & 0 \\
\hline & & 9,5 & 0,9 & 86,0 & 3,5 \\
\hline \multicolumn{6}{|l|}{ Tareas de casa $(n=5.101)$} \\
\hline Moderada & 20,6 & 4,5 & 0,5 & $14,6^{\mathrm{a}}$ & $1 \mathrm{a}$ \\
\hline Grave & 35,0 & $6,7^{\mathrm{b}}$ & $0,7^{\mathrm{b}}$ & 24,8 & 2,7 \\
\hline \multirow[t]{2}{*}{ Incapaz } & 44,4 & 0 & 0 & 44,4 & 0 \\
\hline & & 11,2 & 1,2 & 83,9 & 3,7 \\
\hline \multicolumn{6}{|l|}{ Cuidar a otros $(n=3.308)$} \\
\hline Moderada & 12,7 & 3,5 & 0,2 & $8,5^{\mathrm{a}}$ & $0,5^{\mathrm{a}}$ \\
\hline Grave & 27,1 & $8,1^{\mathrm{b}}$ & $0,3^{\mathrm{b}}$ & 16,9 & 1,9 \\
\hline \multirow[t]{2}{*}{ Incapaz } & 60,1 & 0 & 0 & 60,1 & 0 \\
\hline & & 11,6 & 0,5 & 85,5 & 2,5 \\
\hline
\end{tabular}

Los porcentajes se han calculado tomando como denominador el $\mathrm{n}^{\circ}$ de personas discapacitadas en cada actividad.

a: Porcentaje de personas excluidas con el criterio de disc. residual severa respecto a dependencia más incapacidad.

b: Porcentaje de personas excluidas con el criterio de dependencia más incapacidad respecto a disc. residual severa.

alternativamente, se determinara mediante dependencia más incapacidad se excluiría a un $4 \%(3,4+0,6)$ de personas con discapacidad residual severa. Globalmente, y como reflejan más claramente las figuras $1,2,3$, y 4, dependencia más incapacidad excluye a una mayor proporción de personas con discapacidad subyacente en movilidad y discapacidad residual severa excluye a una mayor proporción de personas con discapacidad subyacente en actividades domésticas y sobre todo en autocuidados. La comparación entre dependencia (sin incluir, por definición, in- 
Figura 1

Discapacidad residual severa y dependencia más incapacidad según la distribución de la ayuda en movilidad interior

\begin{tabular}{|c|c|c|c|c|c|}
\hline 18,8 & 12 & \begin{tabular}{l|l}
5 & 5,5 \\
\end{tabular} & 8,7 & 35,4 & $2, \beta \quad 11,9$ \\
\hline \multicolumn{2}{|c|}{30,8} & 10,5 & & 44,1 & 14,7 \\
\hline \multicolumn{2}{|c|}{$\mathrm{S} / \mathrm{t}$} & $\mathrm{T}$ & & $P$ & Tу $P$ \\
\hline
\end{tabular}

Posiciones

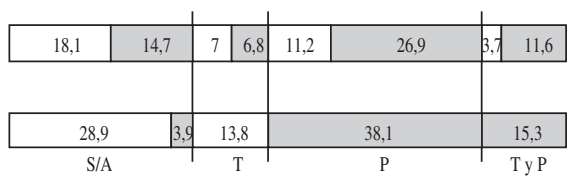

Transferencia

\begin{tabular}{|c|c|c|c|c|c|c|c|}
\hline 12,2 & 12,2 & 14,1 & 11,6 & 7 & 20,1 & 4,9 & 18 \\
\hline 19,2 & 5,2 & & & & 27,1 & & 22,9 \\
\hline S/A & & & & & $\mathrm{P}$ & & T y $P$ \\
\hline
\end{tabular}

\section{Andar dentro}

S/A: Sin Ayuda. T: Con ayuda técnica. P: Con ayuda personal. T y P: Con ayuda técnica y personal.

$\square$ En blanco: independiente o sin discapacidad residual severa. $\square$ : dependiente o con discapacidad residual severa.

Para cada actividad, la barra superior representa discapacidad residual severa y la inferior dependencia más incapacidad.

\section{Figura 2}

Discapacidad residual severa y dependencia más incapacidad según la distribución de la ayuda en autocuidados

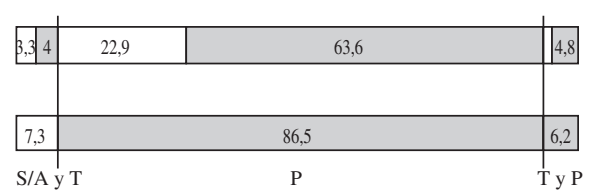

Aseo

\begin{tabular}{|c|c|c|c|c|}
\hline 4 & 8,1 & 11,5 & 59,5 & 15 \\
\hline & \multicolumn{3}{|c|}{} \\
\hline 11,5 & 6 & 71 & 16,9 \\
\hline \multicolumn{2}{|c|}{ S/A y T } & P & T y P
\end{tabular}

Uso WC

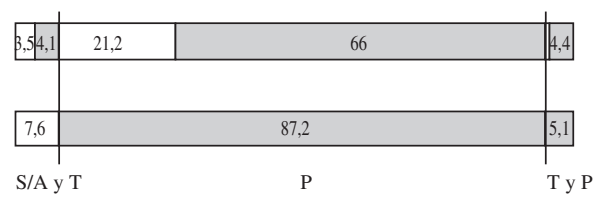

\section{Vestirse}

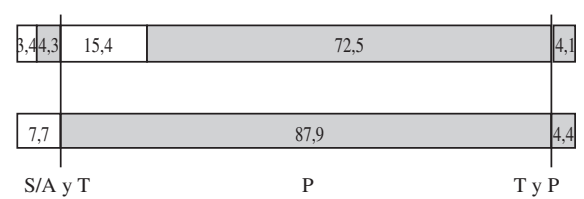

Comer y beber

S/A: Sin Ayuda. T: Con ayuda técnica. P: Con ayuda personal. T y P: Con ayuda técnica y personal.

$\square$ En blanco: independiente o sin discapacidad residual severa. $\square$ : dependiente o con discapacidad residual severa.

Para cada actividad, la barra superior representa discapacidad residual severa y la inferior dependencia más incapacidad. 
Figura 3

Discapacidad residual severa y dependencia más incapacidad según la distribución de la ayuda en movilidad exterior

\begin{tabular}{|c|c|c|c|c|c|}
\hline 23,6 & 20,2 & \begin{tabular}{l|l|}
6,5 & 6,3 \\
\end{tabular} & 10,5 & 19,3 & $\begin{array}{lll}3,5 & 10,1\end{array}$ \\
\hline 25,7 & 18,1 & 12 & & 29,8 & 13,6 \\
\hline
\end{tabular}

Uso de transporte

\begin{tabular}{|c|c|c|c|c|c|c|}
\hline 12,4 & 22,2 & \multicolumn{2}{|r|}{9,3} & $\begin{array}{l}9,3 \\
\end{array}$ & 18,3 & 4,4 \\
\hline 23, & 11,5 & & & & 27,6 & 17,6 \\
\hline & & & & & $\mathrm{P}$ & Ту P \\
\hline
\end{tabular}

Andar fuera

S/A: Sin Ayuda. T: Con ayuda técnica. P: Con ayuda personal. T y P: Con ayuda técnica y personal.

$\square$ En blanco: independiente o sin discapacidad residual severa. $\square$ : dependiente o con discapacidad residual severa.

Para cada actividad, la barra superior representa discapacidad residual severa y la inferior dependencia más incapacidad.

\section{Figura 4}

Discapacidad residual severa y dependencia más incapacidad según la distribución de la ayuda en actividades domésticas

\begin{tabular}{|c|c||c|}
\hline $3,6,2$ & 76,1 & 22 \\
\hline 9,3 & & \\
\hline S/A y T & 87,7 & 3 \\
\hline
\end{tabular}

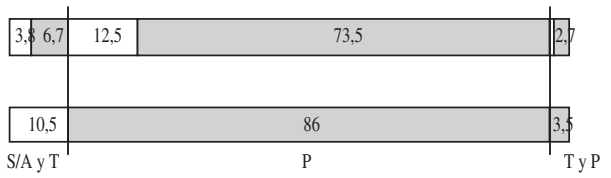

\section{Comidas}

\section{Lavar y planchar}

\begin{tabular}{r|c|c||c|}
\hline $3,46,5$ & 11 & 75,5 & 2, \\
& & & \\
\hline 9,9 & & 36,5 & 3,0 \\
S/A y T & $\mathrm{P}$ & Ty $P$
\end{tabular}

\begin{tabular}{|c|c|c|c|}
\hline \begin{tabular}{l|l|}
5 & 7,4
\end{tabular} & 14,6 & 69,3 & 2,7 \\
\hline 12,4 & & 83,9 & 3,7 \\
\hline
\end{tabular}

\section{Comprar}

\section{Tareas de casa}

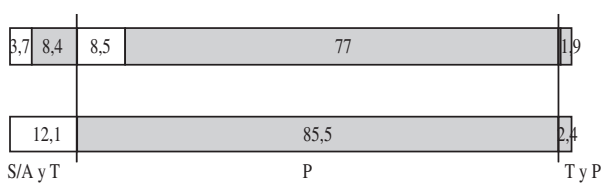

\section{Cuidar a otros}

S/A: Sin Ayuda. T: Con ayuda técnica. P: Con ayuda personal. T y P: Con ayuda técnica y personal.

$\square$ En blanco: independiente o sin discapacidad residual severa. $\square$ : dependiente o con discapacidad residual severa.

Para cada actividad, la barra superior representa discapacidad residual severa y la inferior dependencia más incapacidad. 
Tabla 4

Concordancia entre discapacidad residual severa y los indicadores de dependencia: Coeficiente Kappa, proporción de acuerdo e índices de prevalencia y sesgo

\begin{tabular}{|c|c|c|c|c|c|c|c|c|}
\hline & \multicolumn{4}{|c|}{ Dependencia y disc. resid. severa } & \multicolumn{4}{|c|}{ Dep.+ incapacidad y disc. resid. severa } \\
\hline & Kappa & Po & I.P. & I.S. & Kappa & Po & I.P. & I.S. \\
\hline \multicolumn{9}{|l|}{ M. interior } \\
\hline Posiciones & 0,71 & 0,92 & 0,65 & 0,01 & $=$ & $=$ & $=$ & $=$ \\
\hline Transferencia & 0,57 & 0,84 & 0,51 & 0,03 & 0,63 & 0,86 & 0,49 & 0,01 \\
\hline Andar dentro & 0,60 & 0,87 & 0,58 & 0,04 & 0,66 & 0,89 & 0,56 & 0,02 \\
\hline \multicolumn{9}{|l|}{ Autocuidados } \\
\hline Aseo & 0,76 & 0,91 & 0,45 & 0,07 & $=$ & $=$ & $=$ & $=$ \\
\hline Uso WC & 0,86 & 0,97 & 0,72 & 0,01 & $=$ & $=$ & $=$ & $=$ \\
\hline Vestirse & 0,80 & 0,93 & 0,53 & 0,05 & $=$ & $=$ & $=$ & $=$ \\
\hline Comer, beber & 0,87 & 0,98 & 0,82 & 0,01 & $=$ & $=$ & $=$ & $=$ \\
\hline \multicolumn{9}{|l|}{ M. exterior } \\
\hline Andar fuera & 0,32 & 0,67 & 0,21 & 0,13 & 0,50 & 0,75 & 0,13 & 0,04 \\
\hline Uso de transporte & 0,22 & 0,61 & 0,14 & 0,17 & 0,50 & 0,75 & 0,01 & 0,03 \\
\hline \multicolumn{9}{|l|}{ A. domésticas } \\
\hline Comprar & 0,80 & 0,90 & 0,03 & 0,03 & $=$ & $=$ & $=$ & $=$ \\
\hline Preparar comida & 0,84 & 0,93 & 0,30 & 0,02 & $=$ & $=$ & $=$ & $=$ \\
\hline Lavar, planchar & 0,80 & 0,90 & 0,16 & 0,03 & $=$ & $=$ & $=$ & $=$ \\
\hline Cuidados casa & 0,73 & 0,86 & 0,00 & 0,05 & $=$ & $=$ & $=$ & $=$ \\
\hline Cuidar a otros & 0,85 & 0,93 & 0,34 & 0,00 & $=$ & $=$ & $=$ & $=$ \\
\hline
\end{tabular}

El signo = (en la parte derecha de la tabla) significa que el valor es el mismo que el de la celdilla correspondiente al de la parte izquierda de la tabla (dependencia y discapacidad residual severa). Po es proporción de acuerdo. I.P. es índice de prevalencia. I.S. es índice de sesgo.

capacidad) y discapacidad residual severa sólo es relevante en las cuatro actividades, de movilidad, en las que existe incapacidad sin dependencia, y en esos casos las diferencias se agudizan. Los valores de acuerdo Kappa (tabla 4) entre los dos indicadores de dependencia y discapacidad residual severa reflejan que el error global de clasificación existente excluye que los indicadores sean considerados como intercambiables: la concordancia es sustancial (entre 0,6 y 0,8 ) pero menos que casi perfecta (inferior a 0,8) para la mayoría de las actividades, y en las de movilidad exterior sólo es moderada (o menos que moderada, como sucede con el indicador de dependencia en las cuatro actividades donde hay discapacidad subyacente sin dependencia). En la interpre- tación de Kappa hay que considerar los altos índices de prevalencia en algunas ABVD: lo disminuyen; los índices de sesgo, sin embargo, son despreciables salvo ligeramente en dos casos que implican a la movilidad exterior. Por otra parte, las correlaciones tetracóricas (tabla 5) entre los indicadores de discapacidad y solicitud insatisfecha de ayuda siguen un patrón y magnitud similares a lo largo de las 14 AVD: los valores son más altos con las tareas más sencillas y viceversa, y hay una ligera tendencia a una mayor asociación con dependencia más incapacidad que con discapacidad residual severa.

En la tabla 6 se observa la estructura dimensional de los indicadores de dependencia y de discapacidad residual severa. 
Tabla 5

Correlaciones tetracóricas entre solicitud insatisfecha de ayuda e indicadores de discapacidad

\begin{tabular}{|c|c|c|c|}
\hline & Dependencia & $\begin{array}{c}\text { Dependencia } \\
+ \\
\text { incapacidad }\end{array}$ & $\begin{array}{c}\text { Discapacidad } \\
\text { residual } \\
\text { severa }\end{array}$ \\
\hline \multicolumn{4}{|l|}{ M. interior } \\
\hline Posiciones & 0,70 & $=$ & 0,67 \\
\hline Transferencia & 0,58 & 0,60 & 0,58 \\
\hline Andar dentro & 0,63 & 0,62 & 0,61 \\
\hline \multicolumn{4}{|l|}{ Autocuidados } \\
\hline Aseo & 0,69 & $=$ & 0,66 \\
\hline Uso WC & 0,81 & $=$ & 0,81 \\
\hline Vestirse & 0,75 & $=$ & 0,70 \\
\hline Comer, beber & 0,86 & $=$ & 0,84 \\
\hline \multicolumn{4}{|l|}{ M. exterior } \\
\hline Andar fuera & 0,38 & 0,37 & 0,42 \\
\hline Uso de transporte & 0,33 & 0,30 & 0,35 \\
\hline \multicolumn{4}{|l|}{ A. domésticas } \\
\hline Comprar & 0,44 & $=$ & 0,41 \\
\hline Preparar comida & 0,60 & $=$ & 0,51 \\
\hline Lavar, planchar & 0,55 & $=$ & 0,48 \\
\hline Cuidados casa & 0,41 & $=$ & 0,37 \\
\hline Cuidar a otros & 0,60 & $=$ & 0,52 \\
\hline
\end{tabular}

El signo = significa que el valor es el mismo que el de la dependencia.

Los dos indicadores con mayor varianza explicada por el primer factor son dependencia más incapacidad y discapacidad residual severa; dependencia no sólo tiene un menor primer factor sino que además retiene tres autovalores mayores de uno, uno más que los otros dos indicadores. Las saturaciones factoriales se distribuyen más homogéneamente en el indicador de dependencia más incapacidad que en los otros dos, y en el caso de dependencia, además, dos ítems tienen saturaciones despreciables. Ambos patrones de saturaciones tan heterogéneos, y que se agrupan por dominios de contenido, son inapropiados para desarrollar una medida agregada simple y parsimoniosa de $\operatorname{los}$ datos $^{24}$. Por último, la ratio entre los dos autovalores mayores de uno favo-
Tabla 6

Análisis factorial de información completa: saturaciones factoriales del modelo unidimensional

\begin{tabular}{|l|c|c|c|}
\hline Actividades & $\begin{array}{c}\text { Dependencia } \\
\text { incapacidad } \\
\mathbf{n = 6 . 5 2 2}\end{array}$ & $\begin{array}{c}\text { Dependencia } \\
\mathbf{n}=\mathbf{6 . 4 5 6}\end{array}$ & $\begin{array}{c}\text { Disc. residual } \\
\text { severa } \\
\mathbf{n}=\mathbf{6 . 3 0 0}\end{array}$ \\
\hline Posiciones & 0,71 & 0,61 & 0,55 \\
\hline Transferencia & 0,73 & 0,57 & 0,55 \\
\hline Andar dentro & 0,76 & 0,59 & 0,63 \\
\hline Aseo & 0,76 & 0,70 & 0,78 \\
\hline Uso Wc. & 0,84 & 0,78 & 0,78 \\
\hline Vestirse & 0,80 & 0,73 & 0,80 \\
\hline Comer y beber & 0,80 & 0,75 & 0,76 \\
\hline Andar fuera & 0,62 & 0,17 & 0,47 \\
\hline Uso transporte & 0,60 & 0,06 & 0,48 \\
\hline Comprar & 0,58 & 0,64 & 0,69 \\
\hline Preparar comida & 0,87 & 0,94 & 0,95 \\
\hline Lavar, planchar & 0,85 & 0,95 & 0,98 \\
\hline Cuidados casa & 0,72 & 0,86 & 0,93 \\
\hline Cuidar a otros & 0,79 & 0,82 & 0,84 \\
\hline Porcentaje & 56,33 & 49,37 & 55,82 \\
\hline Aarianza & $8,37,1,91$ & $7,34,1,88,1,13$ & $8,47,2,18$ \\
\hline
\end{tabular}

rece al indicador de dependencia más incapacidad. Por tanto, dependencia más incapacidad es el indicador agregado con mayor validez interna. Su correlación Spearman con discapacidad residual severa es 0,74 .

\section{DISCUSIÓN}

Por definición, y en conformidad con la literatura $^{8-10}$, la discapacidad subyacente es el indicador más inclusivo de discapacidad. Los indicadores de dependencia y de discapacidad residual severa reúnen porcentajes globales similares de personas con discapacidad subyacente, pero hay notables diferencias a nivel de actividades. La incapacidad es mucho más frecuente en las actividades domésticas que en las de autocuidados, lo que es coherente con la mayor dificultad y complejidad de las pri- 
$\operatorname{meras}^{25}$. La distancia es sensiblemente menor entre la movilidad exterior y la movilidad interior. Muchas personas con discapacidad subyacente no reciben ayuda (son independientes) en movilidad y un porcentaje apreciable de personas incapacitadas en movilidad exterior no reciben ayuda. En contraste, la mayoría de las personas con discapacidad subyacente en autocuidados y actividades domésticas son dependientes y no hay personas incapaces en estas actividades que no reciban ayuda. El indicador de solicitud de ayuda insatisfecha (técnica o personal) ordena a las AVD conforme a su dificultad. Conceptualmente es un indicador, como discapacidad residual severa, de necesidad de asistencia ${ }^{25-27}$; pero las correlaciones entre ambos indicadores, en cada una de las AVD, tienden a ser inferiores a las existentes entre solicitud insatisfecha de ayuda y dependencia más incapacidad, en contra de la expectativa. Dado que indaga sobre solicitud de ayuda, no parece ser suficientemente sensible como medida de necesidad, lo que explicaría que sus estimaciones sean inferiores (menos de la mitad) a las de otros estudios en los que se pregunta sobre necesidad de ayuda, no sobre solicitud de $\operatorname{ayuda}^{27}$.

Desde otra perspectiva complementaria, los datos examinados identifican y caracterizan los dominios de la discapacidad ${ }^{28-30}$ : movilidad interior, autocuidados, movilidad exterior y actividades domésticas. La movilidad permite una graduación más fina de la discapacidad subyacente que las actividades de autocuidados y domésticas, ya que la discapacidad subyacente con ayuda técnica y sin ayuda son frecuentes en movilidad pero no en el resto de AVD, donde discapacidad subyacente es casi equivalente a dependencia. Esto es paralelo, pero más acentuadamente, con lo que sucede en la literatura internacional, pues allí es más frecuente que aquí la ayuda sólo técnica en las $\mathrm{ABVD}^{25,27}$ (en las actividades domésticas casi no son aplicables ${ }^{31}$ ).
Es destacable, en esta línea, la escasa frecuencia de ayudas técnicas en las actividades "uso del water" y "aseo personal" por su mayor contraste con lo que sucede en la literatura internacional ${ }^{8,27}$. La cuestión es importante, ya que se ha demostrado que el uso de ayudas técnicas en estas actividades es responsable de la mitad del bien documentado declinar en dependencia en ABVD en Estados Unidos ${ }^{32}$ (en España, por el contrario, se ha observado un ligero incremento $\left.{ }^{14}\right)$. La relación entre movilidad exterior e interior es jerárquica en todos los indicadores de discapacidad; además, mientras que la mayoría de las personas incapaces en movilidad exterior no reciben ayuda, en la movilidad interior es rara la incapacidad sin ayuda. Los autocuidados, frente a las actividades domésticas, se distinguen por la menor presencia de incapacidad $^{24}$ y por su relación jerárquica entre ambas $^{24,28}$. La estructura jerárquica de las ABVD observada en la EDDES es consistente con la constatada en la literatura, si el indicador es dependencia más incapaci$\operatorname{dad}^{33}$ pero no si es discapacidad residual severa. (La estructura jerárquica de las AIVD es mucho menos sólida, así como también las actividades componentes, que en el caso de la EDDES excluyó las "cognitivas": tomar medicinas, uso del teléfono y manejo del dinero ${ }^{34}$ ).

La discapacidad residual severa, respecto a los dos indicadores de dependencia, excluye a un porcentaje estimable de personas dependientes en actividades domésticas y aún más en autocuidados De esta forma, sin embargo, la discapacidad residual severa permite afinar la graduación de la dependencia y quizás aumentar la especificidad de los elegibles beneficiarios de ayuda domiciliaria ${ }^{10}$. Sin embargo, esta graduación podría carecer de suficiente fiabilidad y credibilidad ${ }^{7}$ y hay evidencia de que estas distinciones pueden ser irrelevantes $^{35}$. No así la distinción entre recibir o no recibir ayuda, personal o técnica ${ }^{25,35}$. A la inversa, dependencia más incapacidad 
excluye a un número considerable de personas con discapacidad residual severa; en el caso de la dependencia la exclusión alcanza también a personas con la máxima discapacidad residual (incapacidad). Como medida sumaria de discapacidad en AVD, dependencia más incapacidad tiene mayor validez interna que la discapacidad residual severa y que la dependencia. En la literatura se han combinado en una sola medida las ABVD y las AIVD usando como indicador dependencia más incapa$\operatorname{cidad}^{9,24}$. No existe literatura en el caso de la discapacidad residual severa: este es el primer estudio y no apoya su empleo como tal medida sumaria.

Un aspecto que vertebra gran parte de los resultados de este estudio radica en las diferencias constatadas entre las actividades de movilidad y las de autocuidados y domésticas. Según Nagi ${ }^{36}$ la movilidad se sitúa en el ámbito de las actividades del organismo como un todo (limitaciones funcionales) mientras que los autocuidados y las actividades domésticas en el de los roles y tareas socialmente definidas (discapacidad). En el lenguaje de la nueva Clasificación Internacional del Funcionamiento, de la Discapacidad y de la Salud (CIF) ${ }^{37}$, la movilidad y las actividades de la vida diaria son dominios del componente de la actividad y la participación. Aunque la OMS ha señalado que es difícil distinguir ambos componentes sobre la base de los dominios implicados, Jette et $a l^{38}$ han mostrado que el componente de la actividad reúne actividades o tareas físicas simples (como las de la movilidad) y que el componente de la participación se refiere a categorías de conductas vitales mucho más complejas (como, por ejemplo ocuparse de las comidas), en consonancia con la distinción que realiza Nagi entre limitaciones funcionales y discapacidad. Si esta interpretación es correcta ¿qué sentido tiene combinar actividad y participación (o en términos de Nagi, limitaciones y discapacidad)? Nuestros datos revelan que la es- tructura subyacente de la ayuda y de la incapacidad distinguen la movilidad de los autocuidados y las actividades domésticas; pero el indicador de dependencia más incapacidad iguala las 14 AVD, precisamente, en el umbral de la dependencia y la incapacidad. Siendo esto así, es coherente que de los tres indicadores sólo dependencia más incapacidad presente una sostenible unidimensionalidad.

Señalamos tres limitaciones. Los resultados no son estrictamente generalizables a la población de personas mayores de 64 años, ya que no se han incorporado los pesos del muestreo. La validez de la selección de las actividades consideradas se fundamenta en que son las que generan la gran mayoría de la ayuda en la EDDES, pero ésta no incluyó las AIVD cognitivas. La ausencia de un patrón oro como criterio de discapacidad sólo ha permitido medir el error de clasificación no diferencial entre indicadores, aunque se ha cualificado este error en base a los tipos de ayuda y a la presencia de incapacidad.

Sugerimos dos líneas de investigación: desagregar este tipo de análisis según edad, género y clase social incorporando los pesos del muestreo, y examinar la validez de un índice de severidad de la discapacidad basado en el indicador de dependencia más incapacidad.

En conclusión, la dependencia más la incapacidad frente a la discapacidad residual severa es un indicador más objetivo, está más contrastado en la literatura, es más homogéneo como medida sumaria y es comparable a la discapacidad residual severa como indicador de solicitud insatisfecha de ayuda. La discapacidad residual severa permite, sin embargo, graduar la dependencia en las actividades domésticas y de autocuidados pero a costa de excluir un porcentaje significativo de personas dependientes en las actividades más básicas. 


\section{BIBLIOGRAFÍA}

1. Guralnik JM, Fried LP, Salive ME. Disability as a public health outcome in the aging population. Annu Rev Public Health. 1996; 17:25-46.

2. Picavet HS, Van den Bos GA. Comparing survey data on functional disability: the impact of some methodological differences. J Epidemiol Community Health. 1996; 50(1):86-93.

3. Freedman VA, Crimmins E, Schoeni RF, Spillman BC, Aykan H, Kramarow E et al. Resolving inconsistencies in trends in old-age disability: report from a technical working group. Demography. 2004; 41(3):417-41.

4. Freedman VA. Implications of asking "ambiguous" difficulty questions: an analysis of the second wave of the asset and health dynamics of the oldest old study. J Gerontol B Psychol Sci Soc Sci 2000; 55(5):S288-97.

5. Avlund K. Methodological challenges in measurements of functional ability in gerontological research. A review. Aging (Milano) 1997; 9(3): 164-74.

6. Instituto Nacional de Estadística. Encuesta sobre Discapacidades, Deficiencias y Estado de Salud 1999. Madrid: Instituto Nacional de Estadística; 2001.

7. Verbrugge LM, Jette AM. The disablement process. Soc Sci Med 1994; 38(1):1-14.

8. Jette AM. How measurement techniques influence estimates of disability in older populations. Soc Sci Med 1994; 38(7):937-42.

9. Saliba D, Orlando M, Wenger NS, Hays RD, Rubenstein LZ. Identifying a short functional disability screen for older persons. J Gerontol A Biol Sci Med Sci 2000; 55(12):M750-56.

10. Jenkins CL, Laditka SB. A comparative analysis of disability measures and their relation to home health care use. Home Health Care Serv Q. 2003; 22(1):21-37.

11. Puga D, Abellán A. El proceso de discapacidad. Un análisis de la Encuesta de Discapacidades, Deficiencias y Estado de Salud. Madrid: Fundación Pfizer; 2004.

12. Instituto Nacional de Estadística. Encuesta sobre Discapacidades, Deficiencias y Estado de Salud. Resultados nacionales detallados. Madrid: Instituto Nacional de Estadística; 2002.
13. Instituto de Mayores y Servicios Sociales. Atención a las personas en situación de dependencia en España: libro blanco. Madrid: Imserso; 2005.

14. Sagardui-Villamor J, Guallar-Castillon P, GarciaFerruelo M, Banegas JR, Rodriguez-Artalejo F. Trends in disability and disability-free life expectancy among elderly people in Spain: 1986-1999. J Gerontol A Biol Sci Med Sci. 2005; 60 (8): 1028-34.

15. Alegre A, Ayuso M, Gillén M, Monteverde M, Pociello E. Tasa de dependencia de la población española no institucionalizada y criterios de valoración de la severidad. Rev Esp Salud Pública. 2005; 79(3):351-63.

16. Albarrán I, Alonso P. Clasificación de las personas dependientes a partir de la Encuesta de Discapacidades, Deficiencias y Estado de Salud de 1999. Rev Esp Salud Pública. 2006; 80(3):349-60.

17. SPSS. [computer sofware]. Versión 12.0. Chicago: SPSS Inc; 1999-2003.

18. Byrt T, Bishop J, Carling JB. Bias, prevalence and kappa. J Clin Epidemiol. 1993; 46:423-29.

19. McDonald RP. Factor analysis and related methods. Hillsdale, NJ: LEA; 1984

20. Embretson SE, Reise SP. Item response theory for psychologists. Mahwah, NJ: LEA; 2000.

21. Van der Heyden PGM, Van Buuren S, Fekkes M, Radder J , Verrips E. Unidimensionality and reliability under Mokken scaling of the Dutch language version of the SF-36. Qual Life Res 2003; 12:189-98.

22. Fleishman JA, Spector WD, Altman BM. Impact of differential item functioning on age and gender differences in functional disability. J Gerontol B Psychol Sci Soc Sci J 2002; 57(5):275-84.

23. Bock RD, Gibbons R, Schilling SG, Muraki E, Wilson DT, Wood R. TESTFACT [computer sofware]. Version 4.0. Lincolnwood, IL: Scientific Software International; 2003.

24. Spector WD, Fleishman JA. Combining activities of daily living with instrumental activities of daily living to measure functional disability. J Gerontol B Psychol Sci Soc Sci 1998; 53(1):S46S57.

25. Verbrugge LM, Sevak P. Use, type, and efficacy of assistance for disability. J Gerontol B Psychol Sci Soc Sci. 2002; 57(6):S366-79. 
26. Agree EM. The influence of personal care and assistive devices on the measurement of disability. Soc Sci Med 1999; 48(4):427-43.

27. Agree EM, Freedman VA. A comparison of assistive technology and personal care in alleviating disability and unmet need. Gerontologist. 2003; 43(3):335-44.

28. Verbrugge LM, Yang LS, Juarez L. Severity, timing, and structure of disability. Soz Praventivmed. 2004; 49(2):110-21.

29. Lindeboom R, Vermeulen M, Holman R, de Haan RJ. Activities of daily living instruments:optimizing scales for neurologic assessments. Neurology. 2003; 60(5):738-42.

30. Katz S. Assessing self-maintenance: Activities of daily living, mobility, and instrumental activities of daily living. J Am Geriatr Soc. 1983; 31 (12): 721-27.

31. Allen SM, Foster A, Berg K. Receiving help at home: the interplay of human and technological assistance. J Gerontol B Psychol Sci Soc Sci. 2001; 56(6):S374-82.

32. Freedman VA, Agree EM, Martin LG, Cornman JC. Trends in the use of assistive techno$\operatorname{logy}$ and personal care for late-life disability,
1992-2001. Gerontologist. 2006; 46(1):12427.

33. Lazaridis EN, Rudberg MA, Furner SE, Cassel CK. Do activities of daily living have a hierarchical structure? An analysis using the longitudinal study of aging. J Gerontol. 1994; 49(2):M47-M51.

34. Johnson RJ, Wolinsky FD. The structure of health status among older adults: Disease, disability, functional limitations and perceived health. J Health Soc Behav. 1993; 34: 105-21.

35. Rodgers W, Miller B. A comparative analysis of ADL questions in surveys of older people. J Gerontol B Psychol Sci Soc Sci. 1997; 52(Special issue):21-36.

36. Nagi SZ. Disability concepts revisited: implications for prevention. En: Pope AM, Tarlov AR , editores. Disability in America: toward a national agenda for prevention. Washington DC: National Academy Press; 1991. p. 309-27.

37. World Health Organization. International Classification of Functioning, Disability and Health: ICF. Geneva: WHO; 2001.

38. Jette AM, Haley SM, Kooyoomjian JT. Are the ICF activity and participation dimensions distinct? J Rehabil Med 2003; 35: 145-49. 